\title{
Pregnancy of unknown location: Evidence-based evaluation and management
}

\author{
Identifying pregnancy of unknown location early \\ with a combination of screening modalities \\ guides management of intrauterine or ectopic pregnancy
}

Iris G. Insogna, MD, and Paula C. Brady, MD

IN THIS

ARTICLE

Evaluating

PUL

this page

Tools for

diagnosis

page 29

Steps in

diagnosis

page 31
CASE Woman with bleeding in early pregnancy A 31-year-old woman (G1P0) presents to the local emergency department (ED) due to bleeding in pregnancy. She reports a prior open appendectomy for ruptured appendix; she denies a history of sexually transmitted infections, smoking, and contraception use. She reports having regular menstrual cycles and trying to conceive with her husband for 18 months without success until now.

The patient reports that the previous week she took a home pregnancy test that was positive; she endorses having dark brown spotting

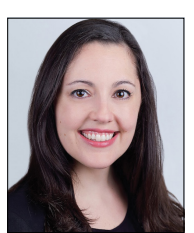

Dr. Insogna is Fellow, Division of Reproductive Endocrinology and Infertility, Department of Obstetrics and Gynecology, Brigham and Women's Hospital, Boston, Massachusetts.

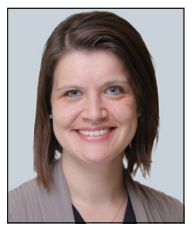

Dr. Brady is Assistant Professor, Department of Obstetrics and Gynecology, Columbia University Irving Medical Center, Columbia University Fertility Center, New York, New York.

The authors report no financial relationships relevant to this article.

doi: 10.12788 /obgm.0021 for the past 2 days but denies pain. Based on the date of her last menstrual period, gestational age is estimated to be 5 weeks and 1 day. Her human chorionic gonadotropin (hCG) level is 1,670 $\mathrm{mlU} / \mathrm{mL}$. Transvaginal ultrasonography demonstrates a normal uterus with an endometrial thickness of $10 \mathrm{~mm}$, no evidence of an intrauterine pregnancy (IUP), normal adnexa bilaterally, and scant free fluid in the pelvis.

\section{Identifying and evaluating} pregnancy of unknown location A pregnancy of unknown location (PUL) is defined by a positive serum hCG level in the absence of a visualized IUP or ectopic pregnancy (EP) by pelvic ultrasonography.

Because of variations in screening tools and clinical practices between institutions and care settings (for example, EDs versus specialized outpatient offices), the incidence of PUL is difficult to capture. In specialized early pregnancy clinics, the rate is $8 \%$ to $10 \%$, whereas in the ED setting, the PUL rate has been reported to be as high as $42 \% .^{1-6}$ While approximately $98 \%$ to $99 \%$ of all pregnancies are intrauterine, only $30 \%$ of PULs will continue to develop as viable ongoing intrauterine gestations. ${ }^{7-9}$ The remainder are revealed 
as failing IUPs or EPs. To counsel patients, set expectations, and triage to appropriate management, it is critical to diagnose pregnancy location as efficiently as possible.

\section{Ectopic pregnancy}

Ectopic pregnancies represent only $1 \%$ to $2 \%$ of conceptions (both spontaneous and through assisted reproduction) and occur most commonly in the fallopian tube, although EPs also can implant in the cornua of the uterus, the cervix, cesarean scar, and more rarely on the ovary or abdominal viscera. ${ }^{10,11}$ Least common, heterotopic pregnancies-in which an IUP coexists with an EP-occur in 1 in 4,000 to 30,000 pregnancies, more commonly in women who used assisted reproduction. ${ }^{11}$

Major risk factors for EP include a history of tubal surgery, sexually transmitted infections (particularly Chlamydia trachomatis), pelvic inflammatory disease, conception with an intrauterine device in situ, and a history of prior EP or tubal surgery, particularly prior tubal ligation; minor risk factors include a history of infertility (excluding known tubal factor infertility) or smoking (in a dose-dependent manner). ${ }^{11,12}$ The concern for an EP is heightened in patients with these risk factors.

Because of the possibility of rupture and life-threatening hemorrhage, EP carries a risk of significant morbidity and mortality. ${ }^{13}$ Ruptured EPs account for approximately $2.7 \%$ of all maternal deaths each year. ${ }^{14}$ When diagnosed sufficiently early in a stable patient, most EPs can be managed medically with methotrexate, a folic acid antagonist. ${ }^{15}$ Ectopic pregnancies also may be managed surgically, and emergency surgery is indicated in women with evidence of EP rupture and intraperitoneal bleeding.

\section{Intrauterine pregnancy}

While excluding EP is critical, it is equally important to diagnose an IUP as expeditiously as possible to avoid inadvertent, destructive intervention. Diagnosis and management of a PUL can involve endometrial aspiration,

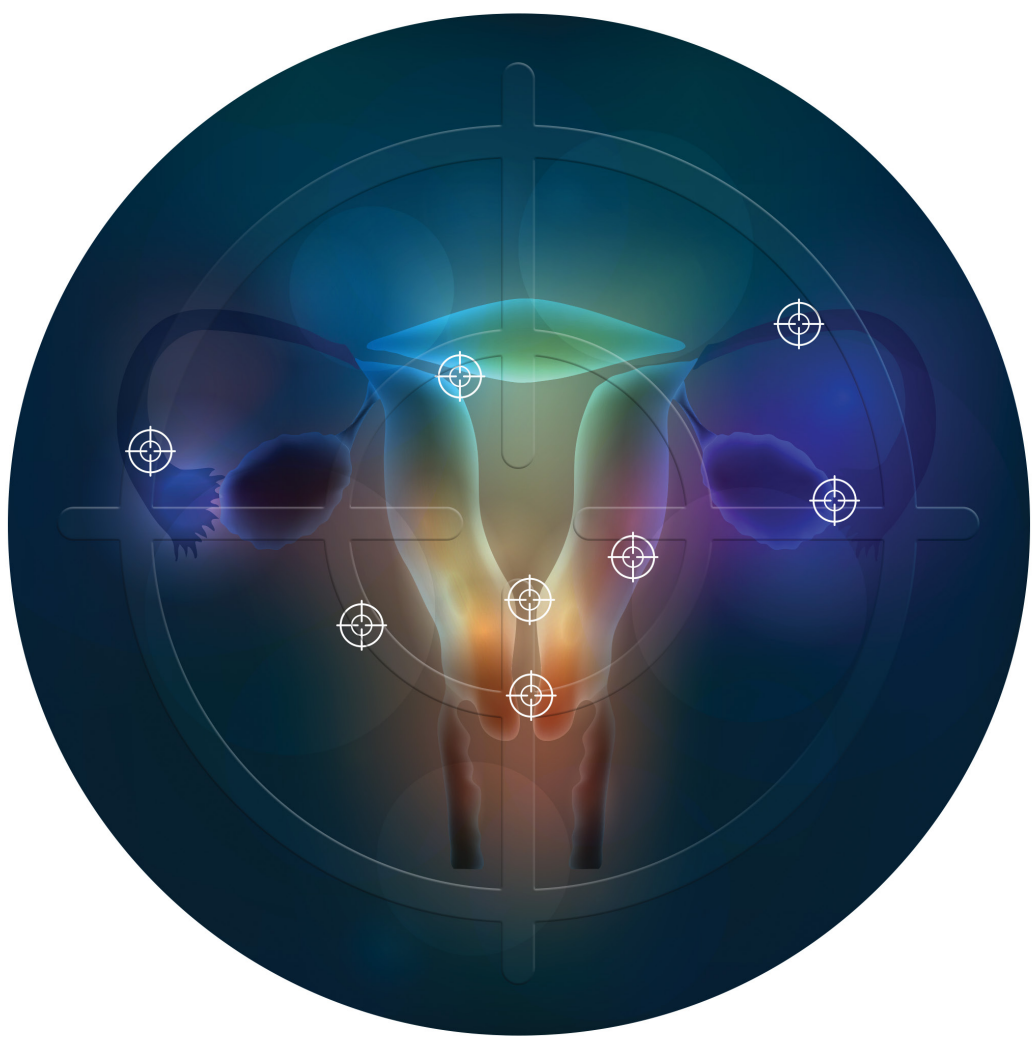

which would interrupt an IUP and should be avoided until the possibility of a viable IUP has been eliminated in desired pregnancies. The inadvertent administration of methotrexate, a known teratogen, to a patient with an undiagnosed viable IUP can result in miscarriage, elective termination, or a live-born infant with significant malformations, all of which expose the administering physician to malpractice litigation..$^{16,17}$

In desired pregnancies, it is essential to differentiate between a viable IUP, a nonviable IUP, and an EP to guide appropriate management and ensure patient safety, whereas exclusion of $\mathrm{EP}$ is the priority in undesired pregnancies.

\section{Tools for diagnosing pregnancy location}

For diagnosing pregnancy location, serial hCG measurement, transvaginal pelvic ultrasonography, and outpatient endometrial 
FAST

TRACK

Recent data

suggest that

the threshold

for minimum

expected

hCG rise for

an ongoing IUP

should be

far lower when

the pregnancy

is desired aspiration are all relevant clinical tools. Pregnancy location can be diagnosed with either direct visualization of an IUP or EP by ultrasonography or with confirmed pathology (chorionic villi or trophoblast cells) from endometrial aspiration (FIGURE). A decline in hCG to an undetectable level following endometrial aspiration also is considered sufficient to diagnose a failed IUP, even in the absence of a confirmatory ultrasonography.

\section{Trending hCG values}

In stable patients with PUL, serum hCG levels are commonly measured at 2-day intervals, ideally for a minimum of 3 values. Conventional wisdom dictates that in viable IUPs, the hCG level should roughly double every 2 days. However, more recent data suggest that the threshold for minimum expected hCG rise for an ongoing IUP should be far lower when the pregnancy is desired.$^{18} \mathrm{~A}$ less conservative cutoff can be considered when a pregnancy is not desired.

In a multisite cohort study of 1,005 women with PUL, a minimum hCG rise of $35 \%$ in 2 days captured the majority of IUPs, with a negative predictive value of $97.2 \%$ for IUP. ${ }^{19}$ Of note, although the cutoff of $35 \%$ was selected to reduce the risk of misdiagnosing an IUP as an EP, $7.7 \%$ of IUPs (and $16.8 \%$ of EPs) were still misclassified, showing that hCG trends must be interpreted in the context of other clinical data, including ultrasonography findings and patient symptoms and history.

A follow-up study demonstrated that hCG rises are lower (but still within this normal range) when the initial hCG value is higher, particularly greater than $3,000 \mathrm{mIU} / \mathrm{mL}^{20}$

Studies show that the rate of spontaneous hCG decline in failing IUPs ranges from $12 \%$ to $47 \%$ in 2 days, falling more rapidly from higher starting hCG values. ${ }^{19,21}$ In a retrospective review of 443 women with spontaneously resolving PUL (presumed to be failing IUPs), the minimum 2-day decline in hCG was $35 \%{ }^{22}$ Any spontaneous hCG decline less than $35 \%$ in 2 days in a PUL should raise physician concern for EP.
Conversely, EPs do not demonstrate predictable hCG trends and can mimic the hCG trends of viable or failing IUPs. Although typically half of EPs present with an increasing hCG value and half present with a decreasing hCG value, the majority (71\%) demonstrate a slower rate of change than either a viable IUP or a miscarriage. ${ }^{11}$ This slower change (plateau) should heighten the clinician's suspicion for an EP.

\section{Progesterone levels}

A progesterone level often is used to attempt to determine pregnancy viability in women who are not receiving progesterone supplementation, although it ultimately has limited utility. While far less sensitive than an hCG value trend, a serum progesterone level of less than 5 to $10 \mathrm{ng} / \mathrm{mL}$ is a rough marker of nonviable pregnancy. ${ }^{23}$

In a large meta-analysis of women with pain and bleeding, $96.8 \%$ of pregnancies with a single progesterone level of less than $10 \mathrm{ng} / \mathrm{mL}$ were nonviable. ${ }^{23}$ When an inconclusive ultrasonography was documented in addition to symptoms of pain and bleeding, $99.2 \%$ of pregnancies with a progesterone level of less than 3.2 to $6 \mathrm{ng} / \mathrm{mL}$ were nonviable.

Progesterone's usefulness in assessing for a PUL is limited: While progesterone levels may indicate nonviability, they provide no indication of pregnancy location (intrauterine or ectopic).

\section{Alternative serologic markers}

Various other reproductive and pregnancyrelated hormones have been investigated for use in the diagnosis of pregnancy location in PULs, including activin A, inhibin $\mathrm{A}$, pregnancy-associated plasma protein $\mathrm{A}$ (PAPP-A), placental-like growth factor, vascular endothelial growth factor, follistatin, and various microRNAs. ${ }^{24,25}$ While research into these biomarkers is ongoing, none have been studied in prospective trials, and they are not for use in current clinical care.

\section{Pelvic ultrasonography}

Pelvic ultrasonography is a crucial part of PUL assessment. Transvaginal ultrasonography 


\section{FIGURE Steps in diagnosing pregnancy of unknown location}

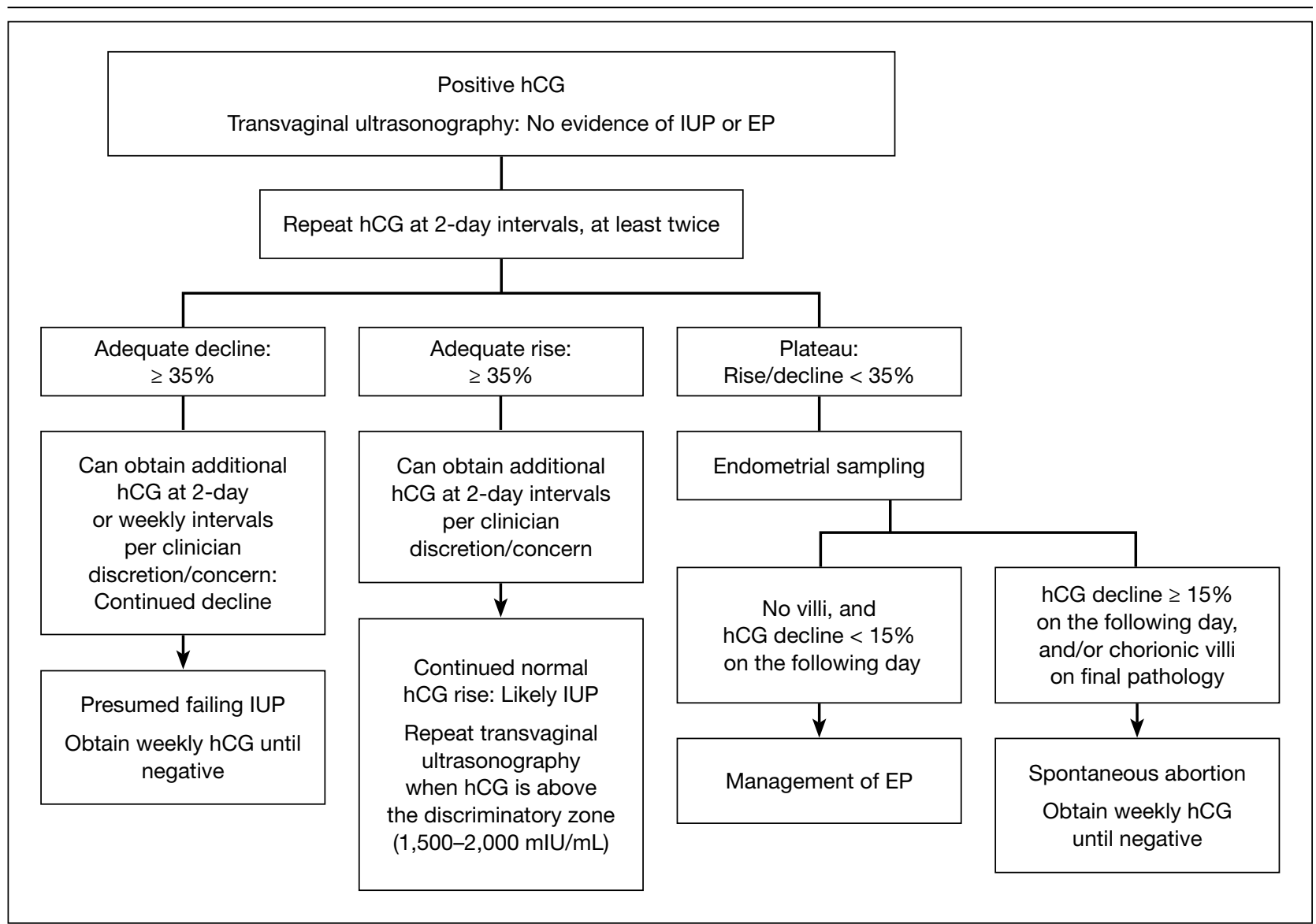

Abbreviations: EP, ectopic pregnancy; hCG, human chorionic gonadotropin; IUP, intrauterine pregnancy.

should be interpreted in the context of the estimated gestational age of the pregnancy and serial hCG values, if available; the patient's symptoms; and the sensitivity of the ultrasonography equipment, which also may be affected by variables that can reduce visualization, such as uterine fibroids and obesity.

The "discriminatory zone" refers to the hCG value above which an IUP should be visualized by ultrasonography. Generally, with an hCG value of 1,500 to $2,000 \mathrm{mIU} / \mathrm{mL}$ or greater, an IUP is expected to be seen with transvaginal sonography.,26 Many exceptions to the discriminatory zone have been reported, however, including multiple pregnancies, which will have a higher hCG value at an earlier gestational age. Even in singleton pregnancies, viable IUPs have been documented as developing from PULs with an elevated initial hCG value as high as $4,300 \mathrm{mIU} / \mathrm{mL}{ }^{27}$ The discriminatory zone may vary among clinical hCG assays, and it also is affected by the quality and modernity of the ultrasonography equipment as well as by the ultrasonography operator's experience and skill. ${ }^{28,29}$

The estimated gestational age, based on either the last menstrual period or assisted reproduction procedure, provides a helpful data point to guide expectations for ultrasonography findings. ${ }^{30}$ Using transvaginal ultrasonography in a normally progressing IUP, a gestational sac-typically measuring 2 to $3 \mathrm{~mm}$ -should be visualized at 5 weeks. ${ }^{15,30} \mathrm{At}$ approximately 5.5 weeks, a yolk sac measuring 3 to $5 \mathrm{~mm}$ should appear. At 6 weeks, an embryo with cardiac activity should be visualized.

In a pregnancy reliably dated beyond 5 weeks, the lack of an intrauterine gestational 
FAST

TRACK

A decline

in $h$ CG levels

of at least $15 \%$

within 24 hours

of endometrial

aspiration

indicates that

a failed IUP is

the most likely

diagnosis

and further

intervention

is not indicated-

although falling

hCG values

should be

monitored

for confirmation sac is suspicious for, but not diagnostic of, an EP. Conversely, the visualization of a gestational sac alone (without a yolk sac) is insufficient to definitively exclude an EP, since a small fluid collection in the endometrium (a "pseudosac") can convincingly mimic the appearance of a gestational sac, and a follow-up ultrasonography should be performed in such cases.

Among patients without ultrasonographic evidence of an IUP, endometrial thickness has been posited as a way to differentiate between IUP and EP. ${ }^{31,32}$ Evidence suggests that an endometrial stripe of at least 8 to $10 \mathrm{~mm}$ may be somewhat predictive of an IUP, while endometrial thickness below $8 \mathrm{~mm}$ is more concerning for EP. This clinical variable, however, has been shown repeatedly to lack sufficient sensitivity and specificity for IUP and should be considered only within the entire clinical context.

\section{Endometrial aspiration}

A persistently abnormal hCG trend and an ultrasonography without evidence of an IUP-particularly with an hCG value above the discriminatory zone and/or with reliable pregnancy dating beyond 5 to 6 weeks-is highly concerning for either a failing IUP or an EP. Once a viable desired IUP is excluded beyond reasonable doubt through these measures, endometrial aspiration to determine pregnancy location is a reasonable next step in PUL management.

Endometrial aspiration can identify a failing IUP by detection of trophoblasts or chorionic villi on pathology and/or by a decline of at least $15 \%$ in hCG, measured on the day of endometrial aspiration and again the following day. Endometrial aspiration is effective even in clinical care settings that do not have rapid pathologic analysis available, as hCG measurement before and within 24 hours after the procedure still can be performed.

Vacuum aspiration (electric or manual) in an operating room or office setting is an effective tool for diagnosing pregnancy location. ${ }^{33,34}$ The use of an endometrial Pipelle for endometrial sampling (typically used for an office endometrial biopsy to diagnose hyperplasia or malignancy) is insufficient for determining pregnancy location. ${ }^{35}$ For all patients managed with this protocol, the hCG value ideally should be followed until it is undetectable, regardless of whether an EP or failing IUP was diagnosed. In rare cases, an EP may be diagnosed by a late plateau in hCG values, following an initial decline consistent with a failing IUP.

Utility for diagnosis. Retrospective studies in patients with PUL following in vitro fertilization have established the utility of outpatient endometrial aspiration with a Karman cannula, followed by a repeat hCG measurement on the day after the procedure. ${ }^{34,36}$ These data demonstrate that between $42 \%$ and $69 \%$ of women were ultimately diagnosed with a failed IUP following endometrial aspiration, thereby sparing them unnecessary exposure to methotrexate.

A decline in hCG levels of at least $15 \%$ within 24 hours after the procedure indicates that a failed IUP is the most likely diagnosis and further intervention is not indicated (although falling hCG values should be monitored for confirmation); confirmatory pathology with chorionic villi or trophoblasts was present in less than half of these women and is not necessary to diagnose a failed IUP. ${ }^{36}$ Women diagnosed with a failed IUP after endometrial aspiration also benefitted from a shorter time to resolution of the nonviable pregnancy by approximately 2 weeks. ${ }^{36}$

Despite the efficacy of endometrial aspiration for the diagnosis of pregnancy location, recent data show that physicians have highly variable approaches to PUL with an hCG plateaued above the discriminatory zone: One-third would first perform endometrial aspiration, while one-third would give methotrexate without further diagnostics. ${ }^{37}$ Academic physicians were 4 times more likely to recommend endometrial aspiration. ${ }^{37}$

Presumed EP. Following endometrial aspiration, if pathology does not confirm an intrauterine gestation and the hCG fails to decline by at least $15 \%$, the diagnosis of a presumed EP is made.

For stable patients with neither evidence of intra-abdominal bleeding nor contraindications to methotrexate (such as blood 
dyscrasias, hepatic or renal insufficiency, active pulmonary or peptic ulcer disease, breastfeeding, or a known intolerance to the medication), methotrexate is recommended for medical management. ${ }^{26}$ Following screening blood work that includes a complete blood count and liver function and renal function tests, the typical methotrexate dose is $50 \mathrm{mg} / \mathrm{m}^{2}$ of body surface area. The singledose regimen entails checking hCG on the day of methotrexate administration and again on days 4 and 7 thereafter. A minimum decline in hCG of $15 \%$ between days 4 and 7 indicates successful treatment; if the hCG decline is below $15 \%$, the patient should receive an additional dose of methotrexate.

There are several published alternative regimens for methotrexate administration, including 2-dose and multidose regimens; the 2-dose protocol (2 doses within 7 days) may be more effective in women with higher hCG $\left(>3,000 \mathrm{mIU} / \mathrm{mL}\right.$ ) or known adnexal mass. ${ }^{26,38}$ Contraindications to methotrexate. In addition to strict medical contraindications to methotrexate, relative contraindications that indicate a higher risk of methotrexate failure include the presence of fetal cardiac activity, EP mass greater than $4 \mathrm{~cm}$, and serum hCG above $5,000 \mathrm{mIU} / \mathrm{mL} .{ }^{26}$ Because of the potential risk of tubal rupture during medical management, relative contraindications also include patient inability to follow up as an outpatient and patient refusal of blood transfusion. ${ }^{26}$ Patients with contraindications to methotrexate, hemodynamic instability, ultrasonographic or clinical evidence of EP rupture, or those electing for surgical management may be managed with laparoscopy. ${ }^{11}$ Discussion of surgical management of EP is beyond the scope of this article.

Follow the hCG level. In patients with a failing IUP or an EP treated with methotrexate or salpingostomy, the hCG level should always be followed until it is negative, usually by weekly measurements once the diagnosis is made. In some cases, the hCG level may plateau after an initial decline, alerting the clinician to failed treatment for a known EP or the need for recategorization of a failed IUP as an EP.

\section{CASE Concluded}

The patient's second and third hCG measurements at 2-day intervals were $1,903 \mathrm{mlU} / \mathrm{mL}$ (14\% rise) and 2,264 $\mathrm{mlU} / \mathrm{mL}$ (16\% rise). At that point, a repeat transvaginal ultrasonography showed no IUP, adnexal mass, or free fluid. The patient was counseled for outpatient endometrial aspiration, which was performed using manual vacuum aspiration. The serum $\mathrm{hCG}$ level on the morning of the procedure was $2,420 \mathrm{mIU} / \mathrm{mL}$. On postprocedure day 1 , the serum hCG level fell to $1,615 \mathrm{mlU} / \mathrm{mL}$, a $33 \%$ decline. The patient was counseled that this decline in hCG indicated a failing IUP. The final pathologic analysis was returned 3 days later, showing no evidence of trophoblasts and chorionic villi. Regardless, the diagnosis of failing IUP remained given the rapid hCG decline; the tissue from the disrupted failing IUP was likely very scant or simply not drawn into the cannula. Serum hCG levels repeated at weekly intervals revealed ongoing decline, and after 4 weeks, the serum hCG was negative.

\section{In summary}

For women diagnosed with PUL, the primary goal is to distinguish an IUP from an EP to reduce the risk of EP rupture through expeditious diagnosis and treatment. In women for whom the pregnancy is desired, distinguishing a viable IUP from a nonviable IUP or an $\mathrm{EP}$ is the more specific goal to avoid intervention on a viable IUP (with methotrexate or endometrial aspiration). In women with abnormal hCG trends and indeterminate ultrasonography results (particularly with a serum hCG above the discriminatory zone), outpatient endometrial aspiration is a highly effective way to determine pregnancy location, which dictates further treatment.

\section{References}

1. Kirk E, Bottomley C, Bourne T. Diagnosing ectopic pregnancy and current concepts in the management of pregnancy of unknown location. Hum Reprod Update. 2014;20:250-261.

2. Kirk E, Condous G, Bourne T. Pregnancies of unknown location. Best Pract Res Clin Obstet Gynaecol. 2009;23: 493-499.

3. Carusi D. Pregnancy of unknown location: evaluation and management. Semin Perinatol. 2019;43:95-100.

4. Banerjee S, Aslam N, Zosmer N, et al. The expectant management of women with early pregnancy of unknown
FAST
TRACK

The primary

goal for women

with PUL is

to distinguish

an IUP from

an EP to reduce

the risk of $E P$

rupture through

expeditious

diagnosis and

treatment 
location. Ultrasound Obstet Gynecol. 1999;14:231-236.

5. Cordina M, Schramm-Gajraj K, Ross JA, et al. Introduction of a single visit protocol in the management of selected patients with pregnancy of unknown location: a prospective study. BJOG. 2011;118:693-697.

6. Mol BW, Hajenius PJ, Engelsbel S, et al. Serum human chorionic gonadotropin measurement in the diagnosis of ectopic pregnancy when transvaginal sonography is inconclusive. Fertil Steril. 1998;70:972-981.

7. Kirk E, Condous G, Van Calster B, et al. Rationalizing the follow-up of pregnancies of unknown location. Hum Reprod. 2007;22:1744-1750

8. Stulberg DB, Cain LR, Dahlquist I, et al. Ectopic pregnancy rates and racial disparities in the Medicaid population, 20042008. Fertil Steril. 2014;102:1671-1676.

9. Zeng MF, Li LM. Frozen blastocyst transfer reduces incidence of ectopic pregnancy compared with fresh blastocyst transfer: a meta-analysis. Gynecol Endocrinol. 2019;35:93-99.

10. Farquhar CM. Ectopic pregnancy. Lancet. 2005;366:583-591.

11. Barnhart KT. Ectopic pregnancy. $N$ Engl J Med. 2009;361: 379-387.

12. Bouyer J, Coste J, Shojaei T, et al. Risk factors for ectopic pregnancy: a comprehensive analysis based on a large casecontrol, population-based study in France. Am J Epidemiol. 2003;157:185-194.

13. Creanga AA, Shapiro-Mendoza CK, Bish CL, et al. Trends in ectopic pregnancy mortality in the United States: 1980-2007. Obstet Gynecol. 2011;117:837-843.

14. Creanga AA, Syverson C, Seed K, et al. Pregnancy-related mortality in the United States, 2011-2013. Obstet Gynecol. 2017;130:366-373.

15. Brady PC. Handbook of Consult and Inpatient Gynecology. Switzerland: Springer International Publishing; 2016.

16. Fridman D, Hawkins E, Dar P, et al. Methotrexate administration to patients with presumed ectopic pregnancy leads to methotrexate exposure of intrauterine pregnancies. J Ultrasound Med. 2019;38:675-684.

17. Nurmohamed L, Moretti ME, Schechter T, et al. Outcome following high-dose methotrexate in pregnancies misdiagnosed as ectopic. Am J Obstet Gynecol. 2011;205:533. e1-533.e3.

18. Barnhart KT, Sammel MD, Rinaudo PF, et al. Symptomatic patients with an early viable intrauterine pregnancy: hCG curves redefined. Obstet Gynecol. 2004;104:50-55.

19. Morse CB, Sammel MD, Shaunik A, et al. Performance of human chorionic gonadotropin curves in women at risk for ectopic pregnancy: exceptions to the rules. Fertil Steril. 2012;97:101-6.e2.

20. Barnhart KT, Guo W, Cary MS, et al. Differences in serum human chorionic gonadotropin rise in early pregnancy by race and value at presentation. Obstet Gynecol. 2016;128:504-511.

21. Barnhart K, Sammel MD, Chung K, et al. Decline of serum human chorionic gonadotropin and spontaneous complete abortion: defining the normal curve. Obstet Gynecol. 2004;104(5, pt 1):975-981

22. Butts SF, Guo W, Cary MS, et al. Predicting the decline in human chorionic gonadotropin in a resolving pregnancy of unknown location. Obstet Gynecol. 2013;122(2 pt 1):337-343.
23. Verhaegen J, Gallos ID, van Mello NM, et al. Accuracy of single progesterone test to predict early pregnancy outcome in women with pain or bleeding: meta-analysis of cohort studies. BMJ. 2012;345:e6077.

24. Senapati S, Sammel MD, Butts SF, et al. Predicting first trimester pregnancy outcome: derivation of a multiple marker test. Fertil Steril. 2016;106:1725-1732.e3.

25. Refaat B, Bahathiq AO. The performances of serum activins and follistatin in the diagnosis of ectopic pregnancy: a prospective case-control study. Clin Chim Acta. 2020;500: 69-74.

26. Practice Committee of American Society for Reproductive Medicine. Medical treatment of ectopic pregnancy: a committee opinion. Fertil Steril. 2013;100:638-644.

27. Doubilet PM, Benson CB. Further evidence agains the reliability of the human chorionic gonadotropin discriminatory level. J Ultrasound Med. 2011;30:1637-1642.

28. Desai D, Lu J, Wyness SP, et al. Human chorionic gonadotropin discriminatory zone in ectopic pregnancy: does assay harmonization matter? Fertil Steril. 2014;101:1671-1674.

29. Ko JK, Cheung VY. Time to revisit the human chorionic gonadotropin discriminatory level in the management of pregnancy of unknown location. $J$ Ultrasound Med. 2014;33:465-471.

30. Doubilet PM, Benson CB, Bourne $\mathrm{T}$, et al; Society of Radiologists in Ultrasound Multispecialty Panel on Early First Trimester Diagnosis of Miscarriage and Exclusion of a Viable Intrauterine Pregnancy. Diagnostic criteria for nonviable pregnancy early in the first trimester. $N$ Engl J Med. 2013;369:1443-1451.

31. Moschos E, Twickler DM. Endometrial thickness predicts intrauterine pregnancy in patients with pregnancy of unknown location. Ultrasound Obstet Gynecol. 2008;32:929934.

32. Ellaithy M, Abdelaziz A, Hassan MF. Outcome prediction in pregnancies of unknown location using endometrial thickness measurement: is this of real clinical value? Eur J Obstet Gynecol Reprod Biol. 2013;168:68-74.

33. Shaunik A, Kulp J, Appleby DH, et al. Utility of dilation and curettage in the diagnosis of pregnancy of unknown location. Am J Obstet Gynecol. 2011;204:130.e1-130.e6.

34. Brady P, Imudia AN, Awonuga AO, et al. Pregnancies of unknown location after in vitro fertilization: minimally invasive management with Karman cannula aspiration. Fertil Steril. 2014;101:420-426.

35. Barnhart KT, Gracia CR, Reindl B, et al. Usefulness of pipelle endometrial biopsy in the diagnosis of women at risk for ectopic pregnancy. Am J Obstet Gynecol. 2003;188:906-909.

36. Insogna IG, Farland LV, Missmer SA, et al. Outpatient endometrial aspiration: an alternative to methotrexate for pregnancy of unknown location. Am J Obstet Gynecol. 2017;217:185.el-185.e9.

37. Parks MA, Barnhart KT, Howard DL. Trends in the management of nonviable pregnancies of unknown location in the United States. Gynecol Obstet Invest. 2018;83:552-557.

38. Alur-Gupta S, Cooney LG, Senapati S, et al. Two-dose versus single-dose methotrexate for treatment of ectopic pregnancy: a meta-analysis. Am J Obstet Gynecol. 2019;221:95-108.e2. 